\title{
HARD SOLAR X-RAY BURSTS
}

\author{
C. DE JAGER \\ (University Observatory 'Sonnenborgh' and Space Research Laboratory, \\ Utrecht, The Netherlands)
}

\section{Hard and Soft X-ray Bursts}

A tentative spectrum of the Sun in the X-ray region is shown in Figure 1 (De Jager, 1967). The quiet Sun emits a measurable spectrum at photon energies below about $3 \mathrm{keV}$. During the occurrence of solar flares an enhancement of the emitted energy, and a hardening of the spectrum is clearly visible. The soft X-ray bursts apparently show a maximum radiation flux at wavelengths of about $10 \AA$.

In the spectral region above about $10 \mathrm{keV}$ hardly any quiet solar radiation is observable. During the occurrence of solar flares hard X-ray bursts are occasionally observed. From the point of view of observational techniques these bursts may be divided in the so-called deka-keV bursts, covering the range $10-200 \mathrm{keV}$, and the deci-MeV bursts in the range between 0.2 and $1 \mathrm{MeV}$. Many deka-keV bursts have been observed during the years $1966-67$ by Winckler et al., by means of the OGO-I and OGO-III satellites (see Arnoldy et al., 1967). The existence of deci-MeV bursts has been doubted various times (see e.g. Chubb et al., 1966). Its reality seems now to be proved (see also De Jager, 1967). However, they may be much rarer than the deka-keV bursts, although it is not yet completely sure that the selection is not observational.

\section{Characteristics of Hard X-ray Bursts}

A sharp distinction between the properties of soft, deka-keV and deci-MeV X-ray bursts can certainly not be made. There is rather a continuous transition between them. Roughly the duration of the bursts seems to decrease with increasing photon energies. It also seems that the number of electrons involved is larger for the sources of soft X-bursts than for those of deci-MeV bursts (see Table 1).

As to their interpretation it is generally assumed that the soft $\mathrm{X}$-bursts are of a quasi-thermal nature; this means that the radiation can be described with the normal radiation laws of a plasma not in local thermal equilibrium. It also seems quite probable that the deci-MeV bursts are of a non-thermal origin. The deka-keV bursts may be in an intermediate state. Part of them may be due to quasi-thermal radiation, others perhaps to non-thermal emission of the plasma. In one case a deka-keV burst 


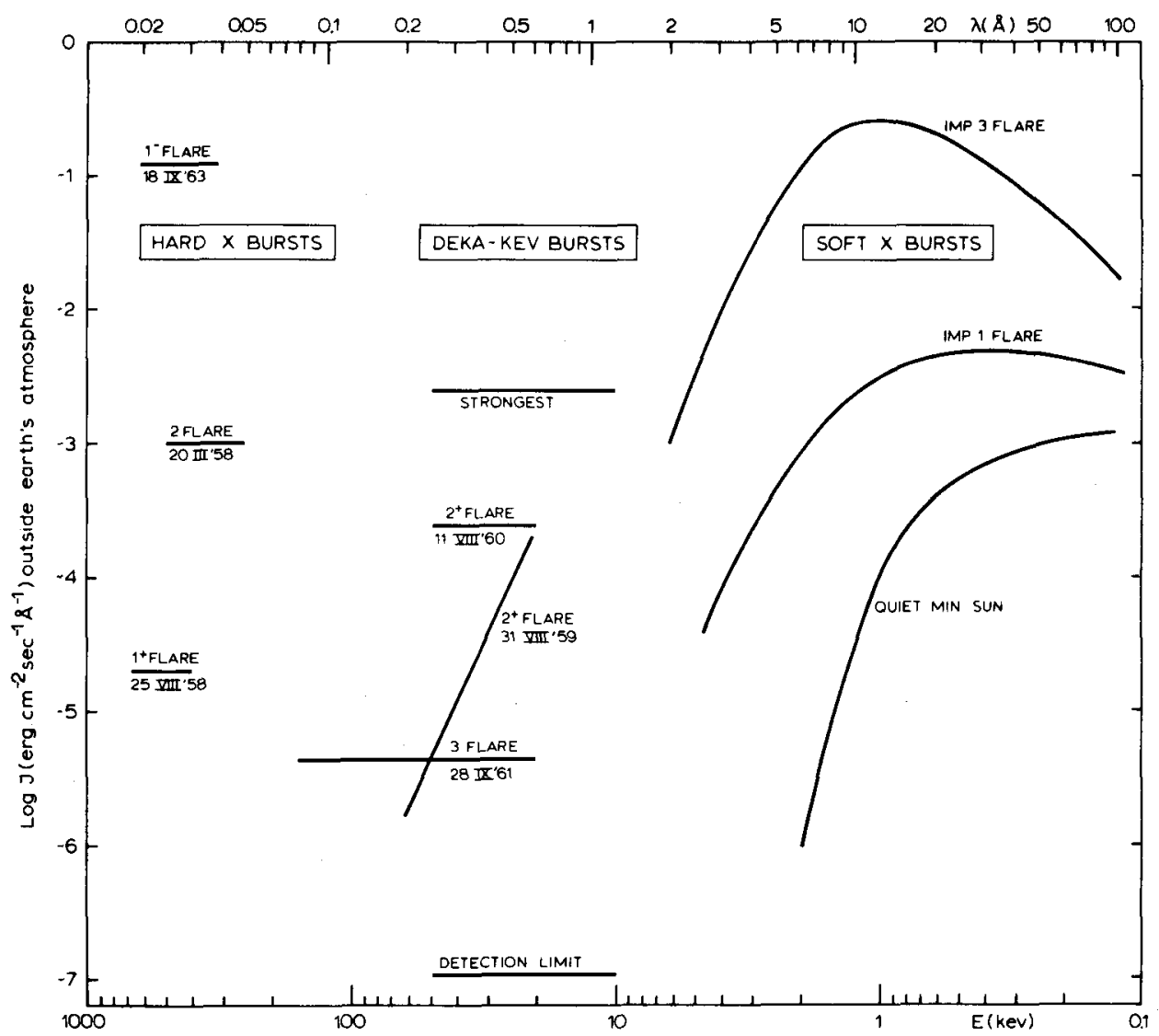

FIG. 1.

\section{Table 1}

Properties of hard $\mathrm{X}$-ray bursts and the associated high-energy flare plasma

soft $X$ bursts

$(E<10 \mathrm{keV})$

deka-keV

(10-200 keV)

hard X bursts

$$
\begin{gathered}
\text { deci-MeV } \\
(0 \cdot 2-1 \mathrm{MeV})
\end{gathered}
$$

few min

as flare

$5-10^{\min }$ $\int N e \mathrm{~d} V$

Origin $\int N e^{2} \mathrm{~d} V$

$10^{47}-10^{49}$

$10^{37}-10^{39 *}$ quasi-thermal

* Assuming $\mathrm{Ne}=10^{10}$. 
had been assumed to consist of a quasi-thermal basis radiation component with a non-thermal burst superimposed on it.

Clearly, the interpretation of these features is still in its infancy, and many more observations should be obtained.

\section{Coordination of Ground and Space Observations in the Investigation of the High-Energy Flare}

We assume that the hard X-ray bursts are due to processes occurring in what we call the high-energy flare plasma: a highly excited transient plasma occurring in connection and together with solar flares. The number of electrons involved in this high-energy plasma ranges between $10^{32}$ (for the weakest deci-MeV bursts) to $10^{39}$ (for the strongest soft X-ray bursts). So, the volume involved may be very small for the high-energy phenomena and seems to be rather large, of the order of that of the sporadic coronal condensations for the plasmas producing the soft X-bursts. Assuming as example an electron density of $10^{10}$ and a thickness of $10^{4} \mathrm{~km}$, one would obtain a projected area, which, if circular, had a diameter ranging between $10^{4}$ and $10 \mathrm{~km}$. A model that is often assumed for the high-energy flare plasma is the one of Takakura and Kai (1966), in which electrons are supposed to be trapped in a magnetic field in an active region.

Important problems to be investigated in order to improve our understanding of the high-energy flare may be the following:

(a) Space observations: complete spectral observations should be obtained of the $\mathrm{X}$-ray bursts in the whole energy range between 100 and $0 \cdot 01 \AA(0 \cdot 1$ and $1000 \mathrm{keV})$. It is not sufficient to have observations in only a small part of the spectrum. In order to understand the acceleration mechanism of the high-energy flare, to define its structure in relation to the other solar features, to investigate the time history of the highenergy flare, it is necessary to have the spectrum of the bursts in this whole energy range, and its variation with time.

(b) Ground-based observations: it seems necessary to obtain radioheliograms with an angular resolution, sufficient to enable the observer to establish the detailed structure of the high-energy radio flare, also in connection to the other solar features and in connection to the high-energy X-ray flare plasma. Spectral observations should be obtained in the whole microwave region, down to wavelengths of about $1 \mathrm{~cm}$, or preferably lower.

\section{References}

Arnoldy, R.L., Kane, S. R., Winckler, J.R. (1967) Solar Phys., 2, 171.

Chubb, T. A., Kreplin, R.W., Friedman, H. (1966) J. geophys. Res., 71, 3611.

De Jager, C. (1967) Solar Phys., 2, 347.

Takakura, T., Kai, K. (1966) Publ. Astron. Soc. Japan, 18, 57. 